\title{
One-Step Synthesis of Saturated Spirocyclic N-Heterocycles with Stannyl Amine Protocol (SnAP) Reagents and Ketones
}

Journal Article

Author(s):

Siau, Woon-Yew; Bode, Jeffrey W.

Publication date:

2014-12-24

Permanent link:

https://doi.org/10.3929/ethz-b-000260428

Rights / license:

In Copyright - Non-Commercial Use Permitted

Originally published in:

Journal of the American Chemical Society 136(51), https://doi.org/10.1021/ja511232b 


\title{
One Step Synthesis of Saturated Spirocyclic N-Heterocycles with SnAP Reagents and Ketones
}

\author{
Woon-Yew Siau and Jeffrey W. Bode \\ Laboratorium für Organische Chemie, Department of Chemistry and Applied Biosciences, ETH-Zürich, 8093 Zürich, Swit- \\ zerland
}

Supporting Information Placeholder

\begin{abstract}
The combination of cyclic ketones and SnAP reagents affords saturated, spirocyclic N-heterocycles under operationally simple reaction conditions. The resulting, $\mathrm{N}$ unprotected spirocyclic amines are in great demand as scaffolds for drug discovery and development. The union of SnAP reagents and acyclic trifluoromethylketones and yields $\alpha-\mathrm{CF}_{3}$ morpholines and piperazines.
\end{abstract}

Saturated, spirocyclic N-heterocycles ${ }^{1,2}$ are regarded as promising scaffolds for drug discovery ${ }^{3}$ and development due to their rigid structures, decreased lipophilicities, and dramatically increased opportunities for fine-tuning the positioning of substituent groups. Despite their recognized advantages, relatively few multifunctional saturated spirocycles are actually available for use due to the lack of synthetic methods to form the spiro-ring junction. Current approaches include insertion of dichlorocarbene $e^{4}$ to give spirolactams or spirolactones and cyclizations of linear precursors. ${ }^{5}$ A major challenge in this area is the preparation of diverse spirocycles by the union of two discrete components. $^{6,7}$

As part of our interest in devising reliable and general synthetic methodologies for the preparation of saturated $\mathrm{N}$ heterocycles, ${ }^{8}$ we recently disclosed SnAP reagents ${ }^{9}$ for the construction of substituted thiomorpholines, morpholines, piperazines, diazepanes, and various medium-ring $\mathrm{N}$ heterocycles from aldehydes. SnAP reagents provide direct routes into $\mathrm{N}$-unsubstituted, fully saturated heterocycles under operationally simple protocols and with outstanding substrate scope. Although we immediately recognized that the use of ketones as substrates would result in the facile formation of saturated spirocyclic compounds (Scheme 1), we approached the implementation of this synthetic route with trepidation as the intermediate ketimines ${ }^{10,11}$ are difficult to prepare and prone to enamine formation. We had also noted that some very sterically hindered aldehydes gave poor yields in the cyclization step, ${ }^{9 b}$ and few reports in the literature on intramolecular cyclizations onto ketimines showed a high preference on 5-exo over 6-endo ring closure. ${ }^{12}$

In this report we document the successful formation of saturated, spirocyclic N-heterocycles, with an emphasis on biand tri-functional derivatives that can serve as valuable platform for further elaboration in drug development efforts. Key to our success was the identification of new conditions for
Scheme 1. One step synthesis of saturated, spirocyclic Nheterocycles with $\mathrm{SnAP}$ reagents and ketones

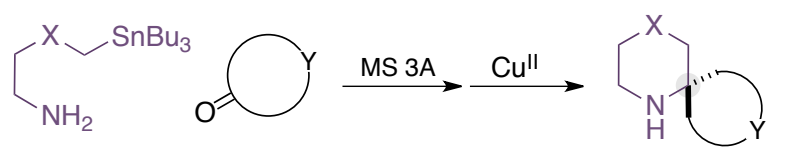

- synthesis of N-heterocyclic spirocycles with SnAP reagents
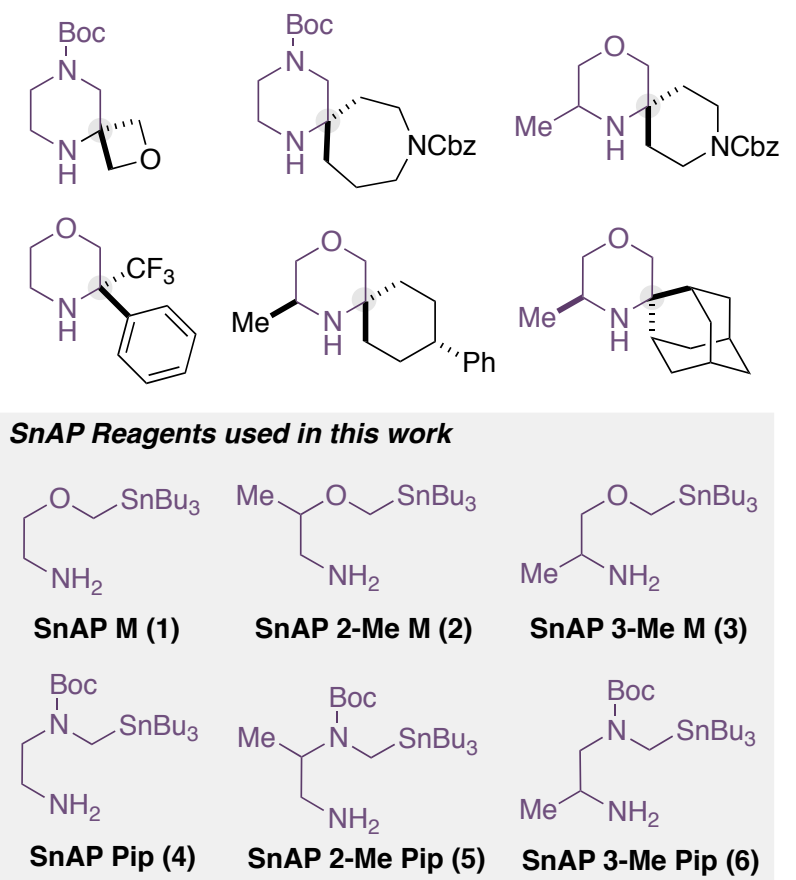

the cyclizations with SnAP reagents on ketone substrates. Although this protocol is not yet suitable for some enolizable ketones, it offers direct access to many of the most interesting spirocyclic structures currently in demand. In many cases, spirocycles that were previously unknown or required lengthy routes $^{13}$ to prepare can now be formed in a single synthetic operation by combination of the appropriate SnAP reagent and ketone.

At the outset of our studies, the efficient formation of the requisite ketimines from ketone substrates and $\mathrm{SnAP}$ reagents required investigation and optimization. Although ketimine formation from certain non-enolizable ketones could be readily achieved, we chose to focus our efforts on cyclic ketones - particularly those bearing an additional functional 
group or substituent - as the resulting products have the greatest utility. We chose $\mathrm{N}-\mathrm{Cbz}$ azetidinone and SnAP 3-MeM 3 as our model substrates and developed conditions for ketimine formation by combining the SnAP reagent (1.0 equiv), the ketone (1.05 equiv), and $\mathrm{MS} 3 \mathrm{~A}$ in refluxing benzene $\left(82^{\circ} \mathrm{C}\right)$ for $12 \mathrm{~h}$. Under our standard conditions for cyclization (1.0 equiv $\mathrm{Cu}(\mathrm{OTf})_{2}, 1.0$ equiv

Scheme 2. One step synthesis of spirocycles from SnAP reagents and ketones.
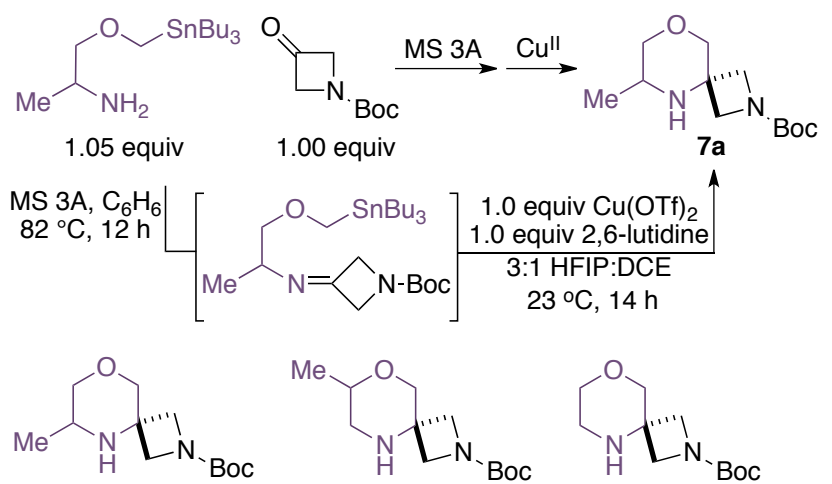

$7 a, 69 \%$

7b, $58 \%$ 7c, $62 \%$<smiles>CC1CN(C(=O)OC(C)(C)C)CC2(CN(C(=O)OC(C)(C)C)C2)N1</smiles><smiles>CC1CNC2(CN(C(=O)OC(C)(C)C)C2)CN1C(=O)OC(C)(C)C</smiles>

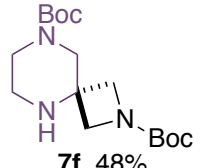<smiles>CC1COCC2(CN(C(=O)c3ccccc3)C2)N1</smiles>

7g, $58 \%$<smiles>CC1COCC2(CCN(C(=O)OC(C)(C)C)CC2)N1</smiles>

7j, $42 \%$<smiles>CC(=O)N1CCC2(CC1)CC(C)N(C(=O)OCc1ccccc1)C2</smiles>

$7 \mathrm{~m}, 40 \%$

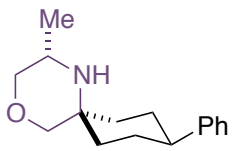

7p, $47 \%$

$(>10: 1 \mathrm{dr})^{b, c}$<smiles>C[C@@H]1COC[C@]2(CCCN(C(=O)OCc3ccccc3)CC2)N1</smiles>

7s, $39 \%$

$(1: 1 d r)^{b}$

Reaction conditions: ketimine (0.50 mmol), $\mathrm{Cu}(\mathrm{OTf})_{2}$ (0.50 mmol), 2,6-lutidine (0.50 mmol), 3:1 HFIP:DCE, 14 h, $23^{\circ} \mathrm{C}$. Yield values refer to isolated yield after purification. ${ }^{b}$ Determined by ${ }^{1} \mathrm{H}$ NMR. ${ }^{c}$ Relative configuration determined by X-ray crystallography. ${ }^{\mathrm{d}}$ Assigned by analogy to $7 \mathbf{p}$. HFIP $=1,1,1,3,3,3$ - hexafluoro-2-propanol. DCE $=1,2$-dichloroethane. Bzh $=$ benzhydryl. Boc = tert-butyloxycarbonyl.

2,6-lutidine, 3:1 HFIP: $\mathrm{ClCH}_{2} \mathrm{CH}_{2} \mathrm{Cl}$ at $23{ }^{\circ} \mathrm{C}$ we could detect desired product formation. Upon further optimization, we found two parameters to be critical for spirocycle formation: 1) the use of an increased amount of HFIP and 2) the integrity of the intermediate ketimines. Under the optimized conditions, the spiro[3.5]-heterocycle $7 \mathbf{a}$ could be obtained in $69 \%$ yield following aqueous workup and column chromatography. We speculated that a higher proportion of HFIP enhances the rate of cyclization.

Encouraged by the successful results using four-membered ring ketones, we explored the synthesis of spiro[5.5]heterocycles with other cyclic ketones. Under the established conditions, 4, 6, and 7-membered cyclic ketones all delivered the expected spirocycles in respectable yield (Scheme 2). A cyclohexanone bearing 4-phenyl substitutent gave a single detectable diastereomer via addition to the equitorial face of the ketimine (7p-r). Substrate specific optimization is likely to be possible; for this study we used a single reaction protocol. The spirocycle formation can be easily used to produce meaningful quantities of the product, even with challenging substrates. For example, amantadine derivative $\mathbf{9}$ was prepared in one step (Scheme 3).

\section{Scheme 3. Synthesis of amantadine 9.}

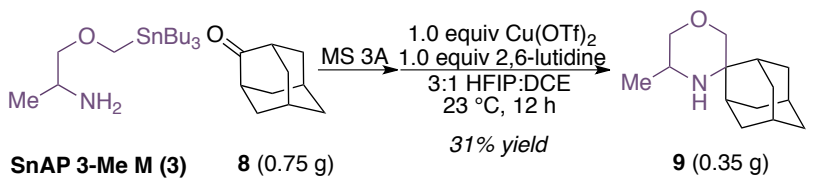

Studies with 5-membered pyrolidinone $\mathbf{1 0}$ and 6membered 3-piperidinone $\mathbf{1 1}$ showed that the integrity of the pre-formed ketimine was crucial for successful cyclization. With the ketimines derived from these substrates - which are prone to enamine formation - we observed large amounts of protodestannylation (Chart 1) along with small amounts of the desired spirocycles. The stable vinylogous amide, derived from 1,3-cyclohexanedione 12, gave only side products and no spirocyclic product was observed. Likewise, only enamines were observed from ketone 13. Attempts to use SnAP reagents that form larger rings, such as SnAP-OA 14, also gave poor yields, as the slower rate of cyclization resulted in large amounts of protodestannylation. In general, we attribute the slightly diminished yields in SnAP reactions with ketone substrates, relative to aldehyde substrates, to the reduced rate of cyclization, leading to competing protodestannylation. Ongoing efforts to improve the stability of the intermediate radical or organocopper intermediate by changing the ligand will address these limitations in the future.

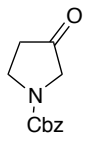

10

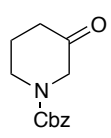

11

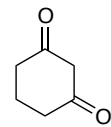

12

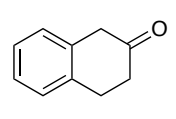

13

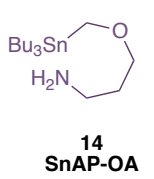

Chart 1. Poor yielding substrates for spirocycle formation due 
to enamine formation or protodestannylation.

Direct condensations of acyclic ketones and SnAP reagents sometimes proved difficult. To overcome this, we devised azido SnAP reagent $\mathbf{1 5}$ to access the required ketimine by the combination of Staudinger and aza-Wittig reactions with polymer-bound triarylphosphine. ${ }^{14,15}$ When 2,2,2trifluoroacetophenone was employed, we achieved full conversion of the ketone to the corresponding ketimines without any trace of triphenylphosphine oxide. Subsequent cyclization proceeded smoothly under the established reaction conditions to afford $\alpha$-trifluoromethyl $\mathrm{N}$-heterocycles 16a16e in acceptable yields (Scheme 4$).{ }^{16}$

Scheme 4. Azido SnAP reagent for $\alpha$-trifluoromethyl Nheterocycles synthesis ${ }^{\mathrm{a}}$

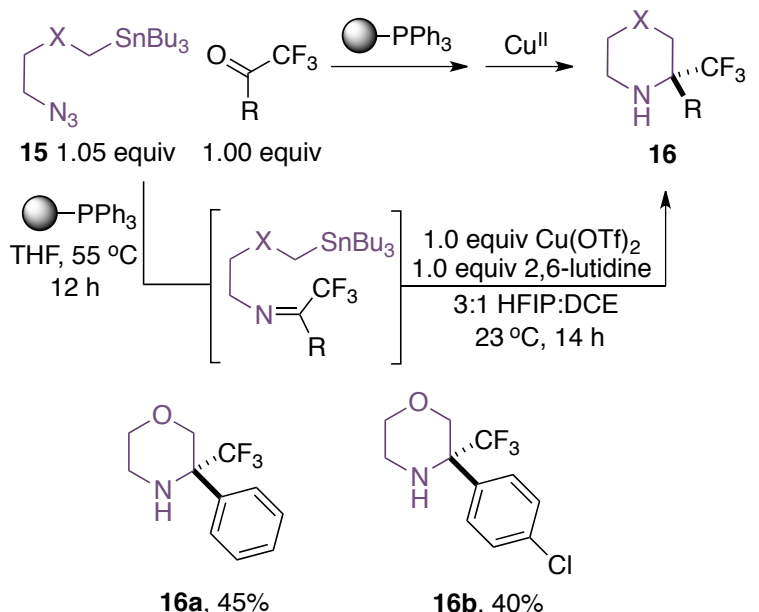

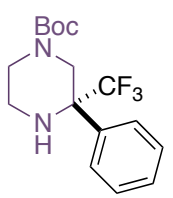

$16 c, 42 \%$

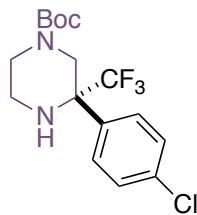

$16 d, 38 \%$

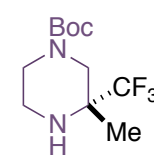

$16 e, 43 \%$
${ }^{a}$ Reaction condition for ketimine formation: azido SnAP reagent (1.05 mmol), ketone $(1.00 \mathrm{mmol})$, polymer-bound triphenyphosphine $(1.10 \mathrm{mmol})$, THF $(10 \mathrm{~mL}), 55{ }^{0} \mathrm{C}, 12 \mathrm{~h}$. Condition for cyclization: ketimine $(1.0 \mathrm{mmol}), \mathrm{Cu}(\mathrm{OTf})_{2}(1.0$ mmol), 2,6-lutidine (1.0 mmol), 3:1 HFIP:DCE, $14 \mathrm{~h}, 23{ }^{\circ} \mathrm{C}$. Yields refer to isolated yields after purification.

In summary, we have shown that the combination of cyclic ketones and SnAP reagents offers single step access to bi- and tri-functional spirocyclic N-heterocycles. It offers an easily recognized synthetic disconnection at the spirojunction, thereby enabling a cross coupling approach to assemble spirocycles from equivalents of their respective monocyclic building blocks. This approach can also be used to prepare trifluoromethyl-substituted morpholines and piperazines from the corresponding ketones. Both of these scaffolds are desired frameworks for modern drug discovery, but their further exploration has been hampered by poor synthetic access and limited routes. SnAP reagents greatly expand the availability of saturated $\mathrm{N}$-heterocycles.

\section{ASSOCIATED CONTENT}

Supporting Information. Experimental procedures and analytical data of all new compounds. This material is available free of charge via the Internet at http://pubs.acs.org.

\section{AUTHOR INFORMATION}

\section{Corresponding Author}

bode@org.chem.ethz.ch

\section{Funding Sources}

This work was supported by the European Research Council (ERC Starting Grant No. 306793 - CASAA and ERC Proof of Concept Grant No. 620214 - SnAP).

\section{ACKNOWLEDGMENT}

We thank LOC MS Service (ETH Zürich) for analyses and Dr. Nils Trapp and Dr. Bernd Schweizer for the acquisition of X-ray structures. We are also grateful to Michael Lüscher, Kimberly Geoghegan, Sheng-Ying Hsieh, and Hidetoshi Noda for helpful discussions. SpiroChem AG is acknowledged for generous gift of oxetan-3-one and 1benzhydrylazetin-3-one.

\section{REFERENCES}

(1) For a recent reviews of spirocyclic N-heterocycles, see: Carreira, E. M.; Fessard, T. C. Chem. Rev. 2014, 114, 8257-8322.

(2) For selected examples of spirocycle synthesis, see: (a) Castaldi, M. P.; Troast, D. M.; Porco, J. A. Org. Lett. 2009, 11, 3362-3365. (b) Badillo, J. J.; Arevalo, G. E.; Fettinger, J. C.; Franz, A. K. Org. Lett. 2011, 13, 418-421. (c) Li, D.-B.; Rogers-Evans, M.; Carreira, E. M. Org. Lett. 2011, 13, 6134-6136. (d) Burkhard, J. A.; Guerot, C.; Knust, H.; Carreira, E. M. Org. Lett. 2012, 14, 66-69. (e) Liu, T.-L.; He, Z.-L.; Tao, H.-Y.; Wang, C.-J. Chem. -Eur. J. 2012, 18, 8042-8046. (f) Kumar, S.; Thornton, P. D.; Painter, T. O.; Jain, P.; Downard, J.; Douglas, J. T.; Santini, C. J. Org. Chem. 2013, 78, 6529-6539.

(3) For selected examples of the use of spirocyclic scaffolds in drug discovery, see: (a) Link, J. O.; Taylor, J. G.; Xu, L.; Mitchell, M.; Guo, H.; Liu, H.; Kato, D.; Kirschberg, T.; Sun, J.; Squires, N.; Parrish, J.; Keller, T.; Y. Z.-Y.; Yang, C.; Matles, M.; Wang, Y.; Wang, K.; Cheng, G.; Tian, Y.; Mogalian, E.; Mondou, E.; Cornpropst, M.; Perry, J.; Desai, M. C. J. Med. Chem. 2014, 57, 2033-2046. (b) Brown, D. G.; Bernstein, P. R.; Griffin, A.; Wesolowski, S.; Labrecque, D.; Tremblay, M. C.; Sylvester, M.; Mauger, R.; Edwards, P. D.; Throner, S. R.; Folmer, J. J.; Cacciola, J.; Scott, C.; Lazor, L. A.; Pourashraf, M.; Santhakumar, V.; Potts, W. M.; Sydserff, S.; Giguère, P.; Lévesque, C.; Dasser, M.; Groblewski, T. J. Med. Chem. 2014, 57, 733-758 (c) Bondada, L.; Rondla, R.; Pradere, U.; Liu, P.; Li, C.; Bobeck, D.; McBrayer, T.; Tharnish, P.; Courcambeck, J.; Halfon, P.; Whitaker, T.; Amblard, F.; Coats, S. J.; Schinazi, R. F. Bioorg. Med. Chem. Lett. 2013, 23, 6325-6330. (d) Efremov, I. V.; Vajdos, F. F.; Borzilleri, K. A.; Capetta, S.; Chen, H.; Dorff, P. H.; Dutra, J. K.; Goldstein, S. W.; Mansour, M.; McColl, A.; Noell, S.; Oborski, C. E.; O'Connell, T. N.; O'Sullivan, T. J.; Pandit, J.; Wang, H.; Wei, B.; Withka, J. M. J. Med. Chem. 2012, 55, 9069-9088. (e) Griffith, D. A.; Dow, R. L.; Huard, K.; Edmonds, D. J.; Bagley, S. W.; Polivkova, J.; Zeng, D.; Garcia-Irizarry, C. N.; Southers, J. A.; Esler, W.; Amor, P.; Loomis, K.; McPherson, K.; Bahnck, K. B.; Préville, C.; Banks, T.; Moore, D. E.; Mathiowetz, A. M.; Menhaji-Klotz, E.; Smith, A. C.; Doran, S. D.; Beebe, D. A.; Dunn, M. F. J. Med. Chem. 2013, 56, 7110-7119. (f) Deng, Y.; Yang, Z.; Shipps Jr, G. W.; Lo, S.-M.; West, R.; Hwa, J.; Zheng, S.; Farley, C.; Lachowicz, J.; van Heek, M.; Bass, A. S.; Sinha, D. P.; Mahon, C. R.; Cartwright, M. E. Bioorg. Med. Chem. Lett. 2013, 23, 791-796. 
(4) Rychnovsky, S. D.; Beauchamp, T.; Vaidyanathan,, R.; Kwan, T. J. Org. Chem. 1998, 63, 6363-6374.

(5) Ruider, S. A.; Müller, S.; Carreira, E. M. Angew. Chem. Int. Ed. 2013, 52, 11908-11911.

(6) Kotha, S.; Deb, A. C.; Lahiri, K.; Manivannan, E. Synthesis 2009, 165-193.

(7) For examples of two-component cross-coupling reactions that afford spirocyclic compounds, see: (a) Barroso, R.; Valencia, R. A., Cabal, M.-P.; Valdés, C. Org. Lett. 2014, 2264-2267. (b) Prandi, C.; Deagostino, A.; Venturello, P.; Occhiato, E. G. Org. Lett. 2005, 7, $4345-4348$.

(8) For general reviews of N-heterocycles synthesis, see: (a) Wolfe, J. P. Synthesis of Heterocycles via Metal-Catalyzed Reactions That Generate One or More Carbon-Heteroatom Bonds; Springer: Berlin, Heidelberg, 2013. (b) Royer, J., Ed. Asymmetric Synthesis of Nitrogen Heterocycles; Wiley-VCH Verlag: Weinheim, 2009. (c) Vo, C.-V.; Bode, J. W. J. Org. Chem. 2014, 79, 2809-2815.

(9) SnAP = Stannyl ( $\underline{\text { Sn })}$ Amine Protocol. (a) Vo, C.-V.; Mikutis, G.; Bode, J. W. Angew. Chem. Int. Ed. 2013, 52, 1705-1708. (b) Luescher, M. U.; Vo, C.-V.; Bode, J. W. Org. Lett. 2014, 16, 12361239; (c) Vo, C.-V.; Luescher, M. U.; Bode, J. W. Nature Chem. 2014, $6,310-314$.

(10) For a review of carbon-centered radicals addition to imines, see: Friestad, G. K. Tetrahedron 2001, 57, 5461-5496.

(11) For selected examples of radical additions to ketimines, see: (a) Miyabe, H.; Yamaoka, Y.; Takemoto, Y. J. Org. Chem. 2005, 70, 33243327. (b) Rossi, B.; Pastori, N.; Clerici, A.; Punta, C. Tetrahedron 2012, 68, 10151-10156

(12) Selected examples on aryl radical cyclization onto aldimines/ketimines, see: (a) Takano, S.; Suzuki, M.; Ogasawara, K.; Heterocycles 1994, 37, 149-152. (b) Tomaszewski, M. J.; Warkentin, J.; Werstiuk, N. H. Aust. J. Chem. 1995, 48, 291-321. (c) Tomaszewski, M. J.; Warkentin, J. Tetrahedron Lett. 1992, 33, 2123-2126. (d) Taka- no, S.; Suzuki, M.; Kijima, A.; Ogasawara, K. Chem. Lett. 1990, 315316.

(13) For examples, see ref. 2 and Kolocouris, N.; Kolocouris, A.; Foscolos, G. B.; Fytas, G.; Neyts, J.; Padalko, E.; Balzarini, J.; Snoeck, R.; Andrei, G.; Clercq, E. D. J. Med. Chem. 1996, 39, 3307-3318.

(14) Staudinger, H.; Meyer, J. Helv. Chim. Acta 1919, 2, 635-646.

(15) For aza-Wittig reactions with solid supported triphenylphosphines, see: (a) Holletz, T.; Cech, D. Synthesis 1994, 8, 789-791. (b) Mahdavi, H.; Amani, J. Tetrahedron Lett. 2009, 50, 5923-5926.

(16) For recent examples of the synthesis of trifluoromethylsubstituted N-heterocycles, see: (a) Dolfen, J.; Kenis, S.; Hecke, K. V.; Kimpe, N. D.; D'hooghe, M. Chem. Eur. J. 2014, 20, 10650-10653. (b) Sánchez-Roselló, M.; Delgado, O.; Mateu, N.; Trabanco, A. A.; Van Gool, M.; Fustero, S. J. Org. Chem. 2014, 79, 5887-5849. (c) Han, J.; Xu, B.; Hammond, G. B. Org. Lett. 2011, 13, 3450-3453.

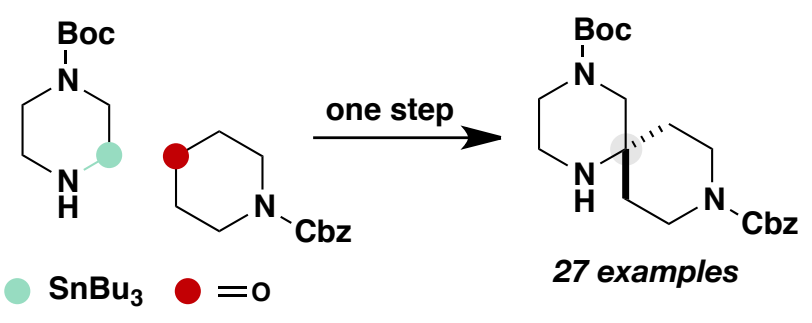


(1)For a recent reviews of spirocyclic N-heterocycles, see: Carreira, E. M.; Fessard, T. C. Chem. Rev. 2014, 114, 8257-8322.

(2) For selected examples of spirocycle synthesis, see: (a) Castaldi, M. P.; Troast, D. M.; Porco, J. A. Org. Lett. 2009, 11, 3362-3365. (b) Badillo, J. J.; Arevalo, G. E.; Fettinger, J. C.; Franz, A. K. Org. Lett. 2011, 13, 418-421. (c) Li, D.-B.; Rogers-Evans, M.; Carreira, E. M. Org. Lett. 2011, 13, 6134-6136. (d) Burkhard, J. A.; Guerot, C.; Knust, H.; Carreira, E. M. Org. Lett. 2012, 14, 66-69. (e) Liu, T.-L.; He, Z.-L.; Tao, H.-Y.; Wang, C.-J. Chem. -Eur. J. 2012, 18, 8042-8046. (f) Kumar, S.; Thornton, P. D.; Painter, T. O.; Jain, P.; Downard, J.; Douglas, J. T.; Santini, C. J. Org. Chem. 2013, 78, 6529-6539.

(3) For selected examples of the use of spirocyclic scaffolds in drug discovery, see: (a) Link, J. O.; Taylor, J. G.; Xu, L.; Mitchell, M.; Guo, H.; Liu, H.; Kato, D.; Kirschberg, T.; Sun, J.; Squires, N.; Parrish, J.; Keller, T.; Y. Z.-Y.; Yang, C.; Matles, M.; Wang, Y.; Wang, K.; Cheng, G.; Tian, Y.; Mogalian, E.; Mondou, E.; Cornpropst, M.; Perry, J.; Desai, M. C. J. Med. Chem. 2014, 57, 2033-2046. (b) Brown, D. G.; Bernstein, P. R.; Griffin, A.; Wesolowski, S.; Labrecque, D.; Tremblay, M. C.; Sylvester, M.; Mauger, R.; Edwards, P. D.; Throner, S. R.; Folmer, J. J.; Cacciola, J.; Scott, C.; Lazor, L. A.; Pourashraf, M.; Santhakumar, V.; Potts, W. M.; Sydserff, S.; Giguère, P.; Lévesque, C.; Dasser, M.; Groblewski, T. J. Med. Chem. 2014, 57, 733-758 (c) Bondada, L.; Rondla, R.; Pradere, U.; Liu, P.; Li, C.; Bobeck, D.; McBrayer, T.; Tharnish, P.; Courcambeck, J.; Halfon, P.; Whitaker, T.; Amblard, F.; Coats, S. J.; Schinazi, R. F. Bioorg. Med. Chem. Lett. 2013, 23, 6325-6330. (d) Efremov, I. V.; Vajdos, F. F.; Borzilleri, K. A.; Capetta, S.; Chen, H.; Dorff, P. H.; Dutra, J. K.; Goldstein, S. W.; Mansour, M.; McColl, A.; Noell, S.; Oborski, C. E.; O’Connell, T. N.; O’Sullivan, T. J.; Pandit, J.; Wang, H.; Wei, B.; Withka, J. M. J. Med. Chem. 2012, 55, 9069-9088. (e) Griffith, D. A.; Dow, R. L.; Huard, K.; Edmonds, D. J.; Bagley, S. W.; Polivkova, J.; Zeng, D.; Garcia-Irizarry, C. N.; Southers, J. A.; Esler, W.; Amor, P.; Loomis, K.; McPherson, K.; Bahnck, K. B.; Préville, C.; Banks, T.; Moore, D. E.; Mathiowetz, A. M.; Menhaji-Klotz, E.; Smith, A. C.; Doran, S. D.; Beebe, D. A.; Dunn, M. F. J. Med. Chem. 2013, 56, 7110-7119. (f) Deng, Y.; Yang, Z.; Shipps Jr, G. W.; Lo, S.-M.; West, R.; Hwa, J.; Zheng, S.; Farley, C.; Lachowicz, J.; van Heek, M.; Bass, A. S.; Sinha, D. P.; Mahon, C. R.; Cartwright, M. E. Bioorg. Med. Chem. Lett. 2013, 23, 791-796.

(4) Rychnovsky, S. D.; Beauchamp, T.; Vaidyanathan,, R.; Kwan, T. J. Org. Chem. 1998, 63, 6363-6374.

(5) Ruider, S. A.; Müller, S.; Carreira, E. M. Angew. Chem. Int. Ed. 2013, 52, 11908-11911.

(6) Kotha, S.; Deb, A. C.; Lahiri, K.; Manivannan, E. Synthesis 2009, 165-193.

(7) For examples of 2-component cross-coupling reactions that afford spirocyclic compounds, see: (a) Barroso, R.; Valencia, R. A., Cabal, M.-P.; Valdés, C. Org. Lett. 2014, 2264-2267. (b) Prandi, C.; Deagostino, A.; Venturello, P.; Occhiato, E. G. Org. Lett. 2005, 7, $4345-4348$.

(8) For general review on N-heterocycles synthesis, see: (a) Wolfe, J. P. Synthesis of Heterocycles via Metal-Catalyzed Reactions That Generate One or More Carbon-Heteroatom Bonds; Springer: Berlin, Heidelberg, 2013. (b) Royer, J., Ed. Asymmetric Synthesis of Nitrogen Heterocycles; Wiley-VCH Verlag: Weinheim, 2009. (c) Vo, C.-V.; Bode, J. W. J. Org. Chem. 2014, 79, 2809-2815.

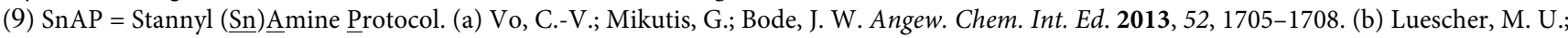
Vo, C.-V.; Bode, J. W. Org. Lett. 2014, 16, 1236-1239; (c) Vo, C.-V.; Luescher, M. U.; Bode, J. W. Nature Chem. 2014, 6, $310-314$.

(10) For review on carbon-centered radicals addition to imines, see: Friestad, G. K. Tetrahedron 2001, 57, 5461-5496.

(11) For selected examples on radical additions to ketimines, see: (a) Miyabe, H.; Yamaoka, Y.; Takemoto, Y. J. Org. Chem. 2005, 70, 3324-3327. (b) Rossi, B.; Pastori, N.; Clerici, A.; Punta, C. Tetrahedron, 2012, 68, 10151-10156

(12)(a) Selected examples on aryl radical cyclization onto aldimines/ketimines, see: (a) Takano, S.; Suzuki, M.; Ogasawara, K.; Heterocycles 1994, 37, 149-152. (b) Tomaszewski, M. J.; Warkentin, J.; Werstiuk, N. H. Aust. J. Chem. 1995, 48, 291-321. (c) Tomaszewski, M. J.; Warkentin, J. Tetrahedron Lett. 1992, 33, 2123-2126. (d) Takano, S.; Suzuki, M.; Kijima, A.; Ogasawara, K. Chem. Lett. 1990, 315-316.

(13) For examples, see ref. 2 and Kolocouris, N.; Kolocouris, A.; Foscolos, G. B.; Fytas, G.; Neyts, J.; Padalko, E.; Balzarini, J.; Snoeck, R.; Andrei, G.; Clercq, E. D. J. Med. Chem. 1996, 39, 3307-3318.

(14) For Staudinger reaction, see: Staudinger, H.; Meyer, J. Helv. Chim. Acta 1919, 2, 635-646.

(15) For aza-Wittig reactions with solid-supported phosphines, see: (a) Holletz, T.; Cech, D. Synthesis 1994, 8, 789-791. (b) Mahdavi, H.; Amani, J. Tetrahedron Lett. 2009, 50, 5923-5926.

(16) (a) Dolfen, J.; Kenis, S.; Hecke, K. V.; Kimpe, N. D.; D’hooghe, M. Chem. -Eur. J. 2014, 20, 10650-10653. (b) Sánchez-Roselló, M.; Delgado, O.; Mateu, N.; Trabanco, A. A.; Van Gool, M.; Fustero, S. J. Org. Chem. 2014, 79, 5887-5849. (c) Han, J.; Xu, B.; Hammond, G. B. Org. Lett. 2011, $13,3450-3453$. 